\title{
On Normal Subgroups of Generalized Hecke Groups
}

\author{
Bilal Demir, Özden Koruoğlu and Recep Sahin
}

\begin{abstract}
We consider the generalized Hecke groups $H_{p, q}$ generated by $X(z)=$ $-\left(z-\lambda_{p}\right)^{-1}, Y(z)=-\left(z+\lambda_{q}\right)^{-1}$ with $\lambda_{p}=2 \cos \left(\frac{\pi}{p}\right)$ and $\lambda_{q}=2 \cos \left(\frac{\pi}{q}\right)$ where $2 \leq p \leq q<\infty, p+q>4$. In this work we study the structure of genus 0 normal subgroups of generalized Hecke groups. We construct an interesting genus 0 subgroup called even subgroup, denoted by $H_{E_{p, q}}$. We state the relation between commutator subgroup $H_{p, q}^{\prime}$ of $H_{p, q}$ defined in [1] and the even subgroup. Then we extend this result to extended generalized Hecke groups $\bar{H}_{p, q}$.
\end{abstract}

\section{Introduction}

In [2] Cangul and Bizim obtained the normal subgroups of genus 0 of Hecke groups $H_{q}$ using the regular map theory. They define a homomorphism of $H_{q}$ to finite triangle groups $A_{4}, S_{4}, A_{5}, C_{n}$ and $D_{n}$. Then the kernel of this homomorphism forms a normal subgroup of genus 0 . For such a subgroup $N$, the quotient group $H_{q} / N$ is isomorphic to one of these finite triangle groups. The signatures of these subgroups presented by permutation method. For all possible situations the number of genus 0 subgrups of $H_{q}$ is calcuated .

The even subgroup $H_{e}\left(\lambda_{q}\right)$ of $H_{q}$ is defined in [3] and [4] for even values of $q$. The structure of $H_{e}\left(\lambda_{q}\right)$ can be obtained by mapping the two elliptic generators of $H_{q}$ to the element of order two in $C_{2}$ and product of them to

Key Words: Generalized Hecke groups, Extended generalized Hecke groups, Genus 0 normal subgroups, Even subgroups.

2010 Mathematics Subject Classification: 20H10, 11F06, 30F35

Received: 07.05.2015

Accepted: 11.06.2015 
identity. It is stated in [4] that the commutator subgroup $H_{q}^{\prime}$, defined in [5], is normal in the even subgroup with index $q$.

Extended Hecke groups $\bar{H}_{q}$ defined in [6] and [7] by adding reflection $R(z)=\frac{1}{\bar{z}}$ to the generators of the Hecke group $H_{q}$. The first commutator subgroup $\bar{H}_{q}^{\prime}$ is normal in $\bar{H}_{q}$ of index 4 for odd $q$, and 8 for even values of $q$. Similar to the Hecke group case, the elements of extended Hecke groups can be classified by odd and even types. Hence the even subgroup $\bar{H}_{e}\left(\lambda_{q}\right)$ of $\bar{H}_{q}$ consists all of the even elements in $\bar{H}_{q}$. For this reason Sahin and Bizim noted that first commutator subgroup $\bar{H}_{q}^{\prime}$ is a normal subgroup of $\bar{H}_{e}\left(\lambda_{q}\right)$, and the index is equal to 4 for even values of $q$.

In this paper, we focus on the normal subgroups of genus 0 of $H_{p, q}$. Firstly, we obtain the structure of these subgroups. We give the number of normal subgroups of genus 0 of generalized Hecke groups $H_{p, q}$, by using the same method in [2] and [3]. In section 4, we give an interesting genus 0 subgroup of generalized Hecke groups, called even subgroup and denoted by $H_{E_{p, q}}$.

Let us begin with background definitions and facts about Hecke groups and generalized Hecke groups.

\section{Motivation and Background Materials}

\subsection{Hecke Groups}

Hecke groups $H(\lambda)$ are included in $P S L(2, \mathbb{R})$, the orientation preserving isometries of the upper half plane $\hat{H}$, and generated by two linear fractional transformations;

$$
T(z)=-\frac{1}{z} \text { and } U(z)=z+\lambda
$$

where $\lambda$ is a fixed positive real number [8]. Let $S=T U$, i.e.,

$$
S(z)=-\frac{1}{z+\lambda} \text {. }
$$

Hecke showed that $H(\lambda)$ is discrete if and only if $\lambda=\lambda_{q}=2 \cos \left(\frac{\pi}{q}\right), q \geq 3$ integer, or $\lambda \geq 2$. We denote the group obtained from the former case $q \geq 3$ integer by $H_{q}=H\left(\lambda_{q}\right)$. Hecke group $H_{q}$ is isomorphic to the free product of two finite cyclic groups of orders 2 and $q$,

$$
H_{q}=<T, S: T^{2}=S^{q}=I>\simeq C_{2} * C_{q} .
$$

Some of the most popular Hecke groups are $H_{3}=\Gamma=P S L(2, \mathbb{Z})$ (the modular group), $H_{4}=H(\sqrt{2}), H_{5}=H\left(\frac{1+\sqrt{5}}{2}\right)$ and $H_{6}=H(\sqrt{3})$. The entries of elements in $H_{q}$ belongs to the ring $\mathbb{Z}\left[\lambda_{q}\right]$. Hence all Hecke groups are subgroups of $\operatorname{PSL}\left(2, \mathbb{Z}\left[\lambda_{q}\right]\right)$. 
Each discrete subgroup $G$ of $P S L(2, \mathbb{R})$ has signature $\left(g ; m_{1}, m_{2}, \ldots, m_{k}\right)$ such that first $r$ of them are finite integers greater than 1 and the rest of them equals to infinity. Then $G$ has presentation;

$$
\left\langle a_{1}, b_{1}, \ldots, a_{g}, b_{g}, x_{1}, x_{2}, \ldots, x_{k} \mid x_{1}^{m_{1}}, x_{2}^{m_{2}}, \ldots, x_{r}^{m_{r}}=x_{1} . x_{2} . \ldots . x_{k} \cdot{ }_{i=1}^{g}\left[a_{i}, b_{i}\right]=1\right\rangle
$$

Here $x_{1}, x_{2}, \ldots, x_{r}$ are elliptic generators and $x_{r+1}, x_{r+2}, \ldots, x_{k}$ are the parabolic generators and $g$ is the genus of the Riemann surface that $G$ acts discontiniously [9]. From this definition the Hecke group $H_{q}$ has signature $(0 ; 2, q, \infty)$.

Normal subgroups of Hecke groups which has genus 0 and related results are studied in [2], [3] and [10]. The calculaton of the total number of these groups, depends on the values of $q$, is made.

The commutator of two elements $A$ and $B$ in a group $G$ defined as $[A, B]=$ $A B A^{-1} B^{-1}$. For any group $G$ one can have an important subgroup generated by all commutators, called the commutator subgroup of $G$. Commutator subgroup is a normal subgroup such that the quotient group is the largest abelien group i.e. let $G$ be a group and $N$ be the commutator subgroup and $H$ be another normal subgroup of $G$ such that $G / H$ is commutative then we have $G / H \leq G / N$. Commutator subgroups of Hecke groups $H_{q}$ studied in [5].

The elements of $H_{q}$ are one of the two forms;

$$
\left(\begin{array}{cc}
a & b \lambda_{q} \\
c \lambda_{q} & d
\end{array}\right) \text { and }\left(\begin{array}{cc}
a \lambda_{q} & b \\
c & d \lambda_{q}
\end{array}\right)
$$

These are the matrix representations of the transformations $\frac{a z+b \lambda_{q}}{c \lambda_{q} z+d}$ and $\frac{a \lambda_{q} z+b}{c z+d \lambda_{q}}$. The former one is called an even type and later one is called an odd.type. Note that when $q=4,6$, then any transformation of one of these forms belongs to $H_{q}$. Even types of elements in $H_{q}$, for even values of $q$, forms a normal subgroup called even subgroup of index 2. Even subgroups and relations to the first commutator subgroup $H_{q}^{\prime}$ of the Hecke group $H_{q}$ is studied in [4].

Extended Hecke groups $\bar{H}_{q}$ defined in [6] and [7] by adding reflection $R(z)=\frac{1}{z}$ to the generators of the Hecke group $H_{q}$. So $H_{q}$ is normal in $\bar{H}_{q}$ of index 2. We have the presentation;

$$
\bar{H}_{q}=<T, S, R: T^{2}=S^{q}=R^{2}=(T R)^{2}=(S R)^{2}=I>\simeq D_{2} *_{C_{2}} D_{q}
$$

The first commutator subgroup $\bar{H}_{q}^{\prime}$ is normal in $\bar{H}_{q}$ of index 4 for odd $q$, and 8 for even values of $q$. Similar to the Hecke group case, the elements of extended Hecke groups can be classified by odd and even types. Hence the 
even subgroup $\bar{H}_{e}\left(\lambda_{q}\right)$ of $\bar{H}_{q}$ consists all of the even elements in $\bar{H}_{q}$. For this reason Sahin and Bizim noted that first commutator subgroup $\bar{H}_{q}^{\prime}$ is a normal subgroup of $\bar{H}_{e}\left(\lambda_{q}\right)$, and the index is equal to 4 for even values of $q[6]$.

\subsection{Generalized Hecke Groups}

In [11] Lehner and Newman determined all faithfull representations of the modular group and extended the result for Hecke groups. They studied the representations of free product of two finite cyclic groups [12]. After these studies Lehner introduced in [13] more general class $H_{p, q}$ of Hecke groups $H_{q}$, by taking

$$
X=\frac{-1}{z-\lambda_{p}} \text { and } V=z+\lambda_{p}+\lambda_{q}
$$

where $2 \leq p \leq q \leq \infty, p+q>4$. Here if we take $Y=X V=-\frac{1}{z+\lambda_{q}}$, then we have the presentation,

$$
H_{p, q}=<X, Y: X^{p}=Y^{q}=I>\simeq C_{p} * C_{q} .
$$

We call these groups as generalized Hecke groups $H_{p, q}$. We know from [13] that $H_{2, q}=H_{q},\left|H_{q}: H_{q, q}\right|=2$, and there is no group $H_{2,2}$. Also, all Hecke groups $H_{q}$ are included in generalized Hecke groups $H_{p, q}$.

From 2.1 we have the signature of $H_{p, q}$ as $(0 ; p, q, \infty)$.

In [14] we defined extended generalized Hecke groups $\bar{H}_{p, q}$, discrete subgroup of isometries of $\hat{H}$, by adding the reflection $R(z)=\frac{1}{\bar{z}}$ to the generators of $H_{p, q}$ with presentation;

$$
\bar{H}_{p, q}=<X, Y, R: X^{p}=Y^{q}=R^{2}=(X R)^{2}=(Y R)^{2}=I>.
$$

The extended generalized Hecke group is isomorphic to the free product of two dihedral groups of orders $p$ and $q$ amalgameted over $C_{2}$.

Calta and Schmidt defined a continued fraction algorithm for groups $H_{3, q}$ to show various properties of this group [15]. They studied Veech groups commensureble with generalized Hecke groups and pseudo-Anasov maps in [16].

We obtained all conjugacy classes of torsion elements of extended generalized Hecke groups $\bar{H}_{p, q}$. We give some properties of index of a subgroup of $\bar{H}_{p, q}$ which has torsion [14]. In [1] we give the abstract group structure of commutator subgroups of $H_{p, q}$ and $\bar{H}_{p, q}$. Also we studied the commutator subgroups and power subgroups of $H_{p, \infty}(\lambda)$ (second kind of generalized Hecke groups with signature $(p, \infty, \infty))$ and $\bar{H}_{p, \infty}(\lambda)$. 


\section{$3 \quad$ Number of genus 0 normal subgroups}

In this section we give the structure of normal subgroups of genus 0 of generalized Hecke groups after that we calculate the total number of these subgroups.

Theorem 1. Let $p, q$ are integers such that $2 \leq p \leq q, p+q>4$.

i) For every divisor $n$ of $q$; the generalized Hecke group $H_{p, q}$ has normal subgroup of genus 0 with signature $\left(0 ; p^{(n)}, \frac{q}{n}, \infty\right)$.

ii) For every divisor $m$ of $p$; the generalized Hecke group $H_{p, q}$ has normal subgroup of genus 0 with signature $\left(0 ; \frac{p}{m}, q^{(m)}, \infty\right)$.

iii) For every divisor $k$ of $(p, q)_{\mathrm{gcd}}$; the generalized Hecke group $H_{p, q}$ has normal subgroup of genus 0 with signature $\left(0 ; \frac{p}{k}, \frac{q}{k}, \infty^{(k)}\right)$.

Proof. Assume that $n \mid q$. Then we can obtain a homomorphism of $H_{p, q}$ to the cyclic group of order $n$, by mapping $X$ to identity and $Y$ to the generator of $C_{n} \simeq(1, n . n)$. This homomorphism gives us a normal subgroup of genus 0 . By permutation method we get the signature $\left(0 ; p^{(n)}, \frac{q}{n}, \infty\right)$.

Similarly we can obtain another homomorphism $H_{p, q} \rightarrow C_{m} \simeq(m, 1, m)$, where $m \mid p$, by mapping $X$ to the generator of $C_{m}$ and $Y$ to identity. So we get a normal subgroup of genus 0 with signature $\left(0 ; \frac{p}{m}, q^{(m)}, \infty\right)$. Since the cyclic group of order $\mathrm{k}$ can be represented as $(k, k, 1)$, we can obtain a homomorphism $H_{p, q} \rightarrow C_{k}$ by mapping $X$ to the generator and $Y$ to inverse of the image of $X$ and we get the subgroup $\left(0 ; \frac{p}{k}, \frac{q}{k}, \infty^{(k)}\right)$. It is important to note that the trivial subgroup $H_{p, q}$ is obtained in all cases because of every positive number is divisible by 1 .

Corollary 1. Let us denote the number of normal genus 0 subgroups of $H_{p, q}$ by $N_{0}$. Then;

$$
N_{0} \geq d(p)+d(q)+d\left((p, q)_{\mathrm{gcd}}\right)+1
$$

where $d$ is the number of divisors greater than one.

Theorem 2. If at least one of $p$ and $q$ is even number, the group $H_{p, q}$ has normal subgroup $N$ of genus 0 such that;

i) $N \simeq\left(0 ; \frac{p}{2}^{(n)}, \frac{q}{n}^{(2)}, \infty^{(n)}\right)$ if $p$ is even and $n \mid q$.

ii) $N \simeq\left(0 ; \frac{p}{n}^{(2)}, \frac{q}{2}^{(n)}, \infty^{(n)}\right)$ if $q$ is even and $n \mid p$

iii) $N \simeq\left(0 ; \frac{p}{2}^{(n)}, \frac{q}{2}^{(n)}, \infty^{(2)}\right)$ if $p$ and $q$ both even.

Proof. By mapping $X$ to element of order 2 and $Y$ to an element of order $n$ in $D_{n} \simeq(2, n, 2)$ where $n \mid q$ and $n>1$. Using the permutation method we get a normal subgroup of genus 0 of $H_{p, q}$ with signature $\left(0 ; \frac{p}{2}^{(n)}, \frac{q}{n}^{(2)}, \infty^{(n)}\right)$. Proof of ii) and iii) can be obtained by presentations of $D_{n}$ as $(n, 2,2)$ and $(2,2, n)$ respectively . 
Remark 1. In view of iii) as in Theorem 2, note that there are infinitely many normal subgroups of genus 0. This type of subgroups as described in iii of Theorem 2 will not be considered in the remaining part of our study. Also the normal subgroup that the quotient group is $D_{2} \simeq(2,2,2)$, is identical in all cases when $p$ and $q$ are both even.

In addition to Theorem 1 and Theorem 2 other normal subgroups of genus 0 can be obtained by thinking the groups $A_{4}, S_{4}$ and $A_{5}$ as homomorphic images of $H_{p, q}$. We can do this under some conditions of $p$ and $q$.

Firstly, let $p$ is even number. Then $H_{p, q}$ has at most 5 more normal genus 0 subgroups.

i) If $3 \mid q$ we can map $H_{p, q}$ to $A_{4}, S_{4}$, and $A_{5}$. Let $A_{4} \simeq<a, b: a^{2}=$ $b^{3}=(a b)^{3}>\simeq(2,3,3)$. By mapping $X$ to $a$ and $Y$ to $b$ we have a homomorphism of $H_{p, q}$ onto $A_{4}$. This gives us a normal subgroup with signature $\left(0 ; \frac{p}{2}^{(6)}, \frac{q}{3}^{(4)}, \infty^{(4)}\right)$. If we map $H_{p, q}$ to $S_{4} \simeq(2,3,4)$ by taking $X$ to the gener-

ator of order 2 and $Y$ to the generator of order 3, we get a normal subgroup with signature $\left(0 ; \frac{p}{2}^{(12)}, \frac{q}{3}^{(8)}, \infty^{(6)}\right)$. Similarly we have another normal subgroup with signature $\left(0 ; \frac{p}{2}{ }^{(30)}, \frac{q}{3}{ }^{(20)}, \infty^{(12)}\right)$ by defining a homomorphism to $A_{5} \simeq(2,3,5)$.

ii) If $4 \mid q$ we can map $H_{p, q}$ to only $S_{4} \simeq(2,4,3)$ and this gives us a normal subgroup with signature $\left(0 ; \frac{p}{2}^{(12)}, \frac{q}{4}^{(6)}, \infty^{(8)}\right)$.

iii) If $5 \mid q$ there is a homomorphism of $H_{p, q}$ to $A_{5} \simeq(2,5,3)$. By permutation method we get a normal subgroup with signature $\left(0 ; \frac{p}{2}{ }^{(30)}, \frac{q}{5}{ }^{(12)}, \infty^{(20)}\right)$.

Secondly if $3 \mid p$ we have four different cases.

i) If $q$ is even there are three homomorphisms of $H_{p, q}$ to $A_{4} \simeq<a, b: a^{3}=$ $b^{2}=(a b)^{3}>\simeq(3,2,3), S_{4} \simeq<a, b: a^{3}=b^{2}=(a b)^{4}>\simeq(3,2,4)$ and $A_{5} \simeq<a, b: a^{3}=b^{2}=(a b)^{5}>\simeq(3,2,5)$ by mapping $X$ to $a$ and $Y$ to $b$. So we have three more normal subgroups with signatures $\left(0 ; \frac{p}{3}{ }^{(4)}, \frac{q}{2}{ }^{(6)}, \infty^{(4)}\right)$, $\left(0 ; \frac{p}{3}^{(6)}, \frac{q}{2}^{(12)}, \infty^{(6)}\right)$ and $\left(0 ; \frac{p}{3}^{(20)}, \frac{q}{2}^{(30)}, \infty^{(12)}\right)$ respectively.

ii) If $3 \mid q$ we can map $H_{p, q}$ to $A_{4} \simeq(3,3,2)$ by taking $X$ and $Y$ to the generators of order 3. This homomorphism gives us a normal subgroup with signature $\left(0 ; \frac{p}{3}{ }^{(4)}, \frac{q}{3}^{(4)}, \infty^{(6)}\right)$.

iii) If $4 \mid q$ we have another homomorphism to $S_{4} \simeq(3,4,2)$ such that $X$ is taken to the generator of order 3 and $Y$ is taken to the generator of order 4 . Then we obtain a normal subgroup with signature $\left(0 ; \frac{p}{3}^{(8)}, \frac{q}{4}^{(6)}, \infty^{(12)}\right)$.

iv) If $5 \mid q$ we can obtain another homomorphism to $A_{5} \simeq(3,5,2)$ by mapping $X$ to the generator of order 3 and $Y$ to the generator of order 5 . Thus we have a normal subgroup with signature $\left(0 ; \frac{p}{3}^{(20)}, \frac{q}{5}{ }^{(12)}, \infty^{(30)}\right)$.

Thirdly if $4 \mid p$ we have two situations; 
i) If $q$ is even there is a homomorphism of $H_{p, q}$ to $S_{4} \simeq(4,2,3)$ such that $X$ is taken to the generator of order 4 and $Y$ is taken to the generator of order 2. This homomorphism gives us a normal subgroup with signature $\left(0 ; \frac{p}{4}^{(6)}, \frac{q}{2}^{(12)}, \infty^{(18)}\right)$.

ii) We have another homomorphism to $S_{4} \simeq(4,3,2)$ by mapping $X$ to the generator of order 4 and $Y$ to the generator of order 3 necessarily $3 \mid q$. So we have a normal subgroup with signature $\left(0 ; \frac{p}{4}^{(6)}, \frac{q}{3}^{(8)}, \infty^{(12)}\right)$.

Finally if $p$ is divisible by 5 we can only map $H_{p, q}$ to $A_{5}$;

i) If $q$ is even the homomorphic image of $H_{p, q}$ is $(5,2,3)$, so we get a normal subgroup with signature $\left(0 ; \frac{p}{5}^{(12)}, \frac{q}{2}^{(30)}, \infty^{(20)}\right)$.

ii) We have another homomorphism to $A_{5} \simeq(5,3,2)$ by mapping $X$ to the generator of order 5 and $Y$ to the generator of order 3 necessarily $3 \mid q$. So we have a normal subgroup with signature $\left(0 ; \frac{p}{5}{ }^{(12)}, \frac{q}{3}{ }^{(20)}, \infty^{(30)}\right)$.

We examined all possible cases. As a conclusion we give the below theorem;

Theorem 3. Let $p, q$ be integers such that $2 \leq p \leq q, p+q>4$. Then there are at least $d(p)+d(q)+d\left((p, q)_{\mathrm{gcd}}\right)+1$ subgroups of genus 0 of generalized Hecke groups $H_{p, q}$. The rest of other possible subgroups, depends on the values of $p$ and $q$, are given as below.

$$
(p, 60)=1\left\{\begin{array}{c}
(q, 60)=1 \\
(q, 20)=1 \text { and } 3 \mid q \\
(q, 12)=1 \text { and } 5 \mid q \\
(q, 4)=1 \text { and } 15 \mid q \\
(q, 60)=2 \\
(q, 20)=2 \text { and } 3 \mid q \\
(q, 15)=1 \text { and } 4 \mid q \\
(q, 5)=1 \text { and } 12 \mid q \\
(q, 4)=2 \text { and } 15 \mid q \\
(q, 12)=2 \text { and } 5 \mid q \\
(q, 3)=1 \text { and } 20 \mid q \\
60 \mid q
\end{array}\right\} \quad d(p)
$$




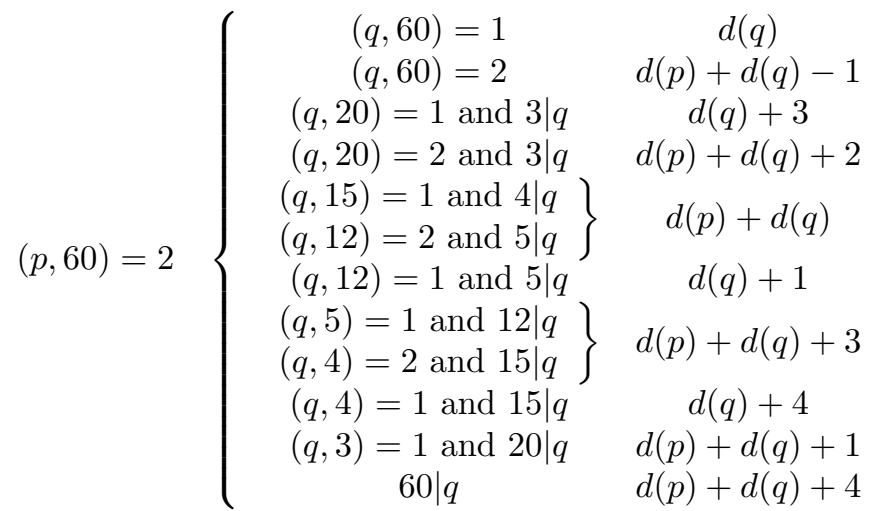$$
(p, 20)=1 \text { and } 3 \mid p \quad\left\{\begin{array}{cc}
\begin{array}{c}
(q, 60)=1 \\
(q, 60)=2
\end{array} & 0 \\
(q, 20)=1 \text { and } 3 \mid q \\
(q, 12)=1 \text { and } 5 \mid q \\
(q, 20)=2 \text { and } 3 \mid q \\
(q, 15)=1 \text { and } 4 \mid q \\
(q, 12)=2 \text { and } 5 \mid q \\
(q, 5)=1 \text { and } 12 \mid q \\
(q, 4)=2 \text { and } 15 \mid q \\
(q, 3)=1 \text { and } 20 \mid q \\
(q, 4)=1 \text { and } 15 \mid q & \\
60 \mid q & d(p)+4 \\
& d(p)+5 \\
& \\
& \\
&
\end{array}\right.
$$

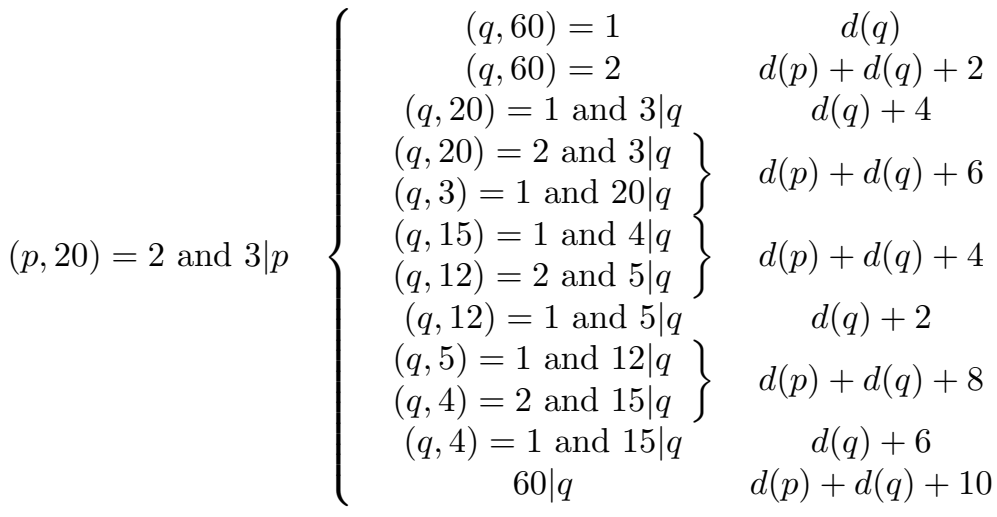




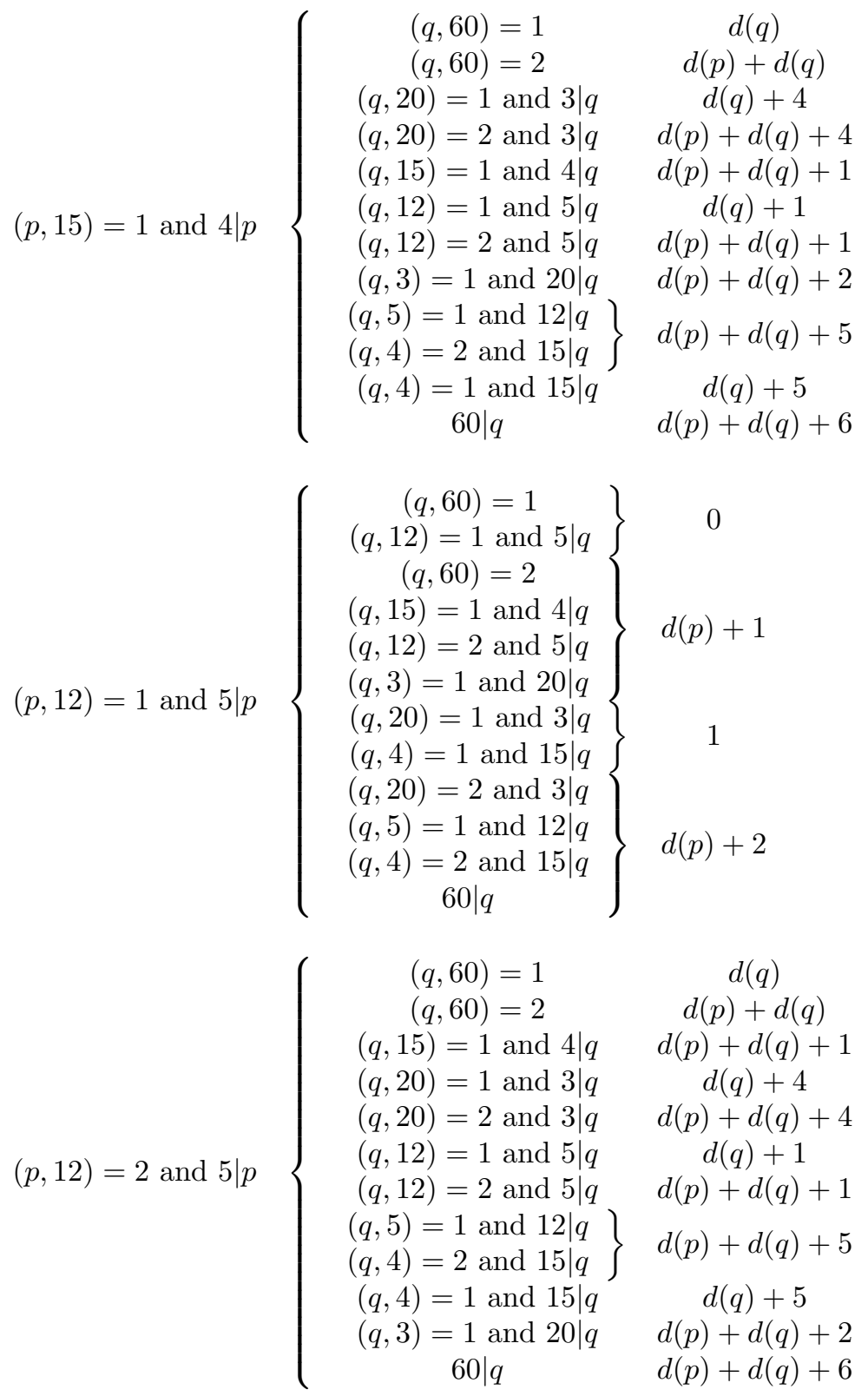




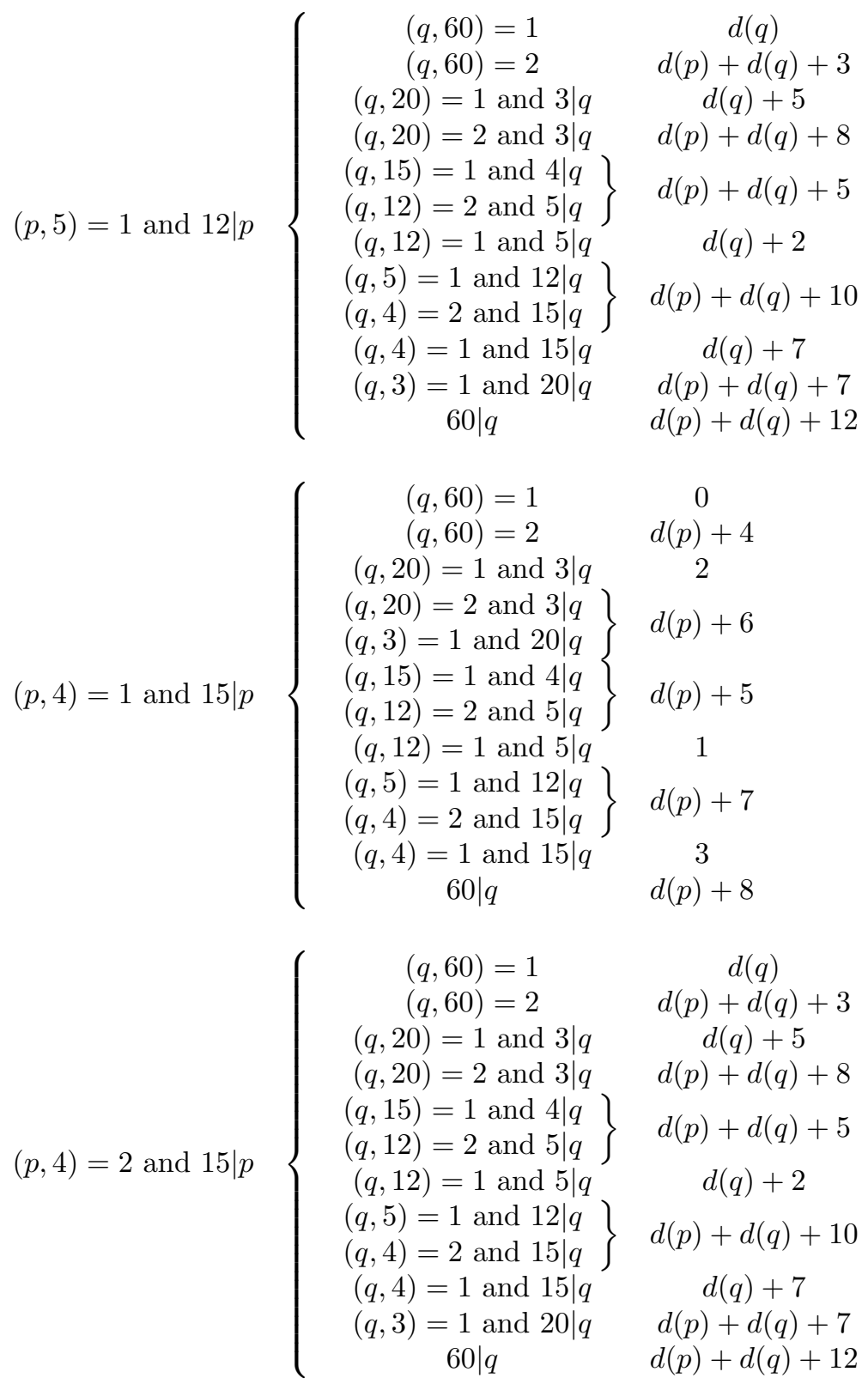




$(p, 3)=1$ and $20 \mid p\left\{\begin{array}{cr}(q, 60)=1 & d(q) \\ (q, 60)=2 & d(p)+d(q)+1 \\ (q, 20)=1 \text { and } 3 \mid q & d(q)+5 \\ (q, 20)=2 \text { and } 3 \mid q & d(p)+d(q)+6 \\ (q, 15)=1 \text { and } 4 \mid q & d(p)+d(q)+2 \\ (q, 12)=1 \text { and } 5 \mid q & d(q)+1 \\ (q, 12)=2 \text { and } 5 \mid q & d(p)+d(q)+2 \\ (q, 5)=1 \text { and } 12 \mid q \\ (q, 4)=2 \text { and } 15 \mid q \\ (q, 4)=1 \text { and } 15 \mid q & d(p)+d(q)+7 \\ (q, 3)=1 \text { and } 20 \mid q & d(p)+d(q)+3 \\ 60 \mid q & d(p)+d(q)+8\end{array}\right.$

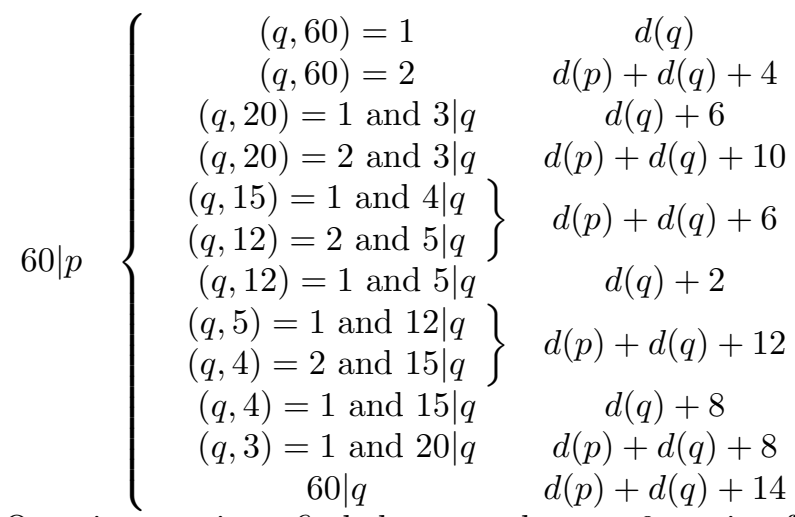

Our aim now is to find the normal genus 0 torsion-free subgroups of generalized Hecke groups. From the above theorem we have the following;

Theorem 4. The normal genus 0 torsion-free subgroups of generalized Hecke groups are given in the table below;

\begin{tabular}{|l|l|l|}
\hline$N$ & $H_{p, q}$ & index \\
\hline$\left(0 ; \infty^{(4)}\right) \triangleleft$ & $H_{2,3}$ & 12 \\
\hline$\left(0 ; \infty^{(6)}\right) \triangleleft$ & $H_{2,3}$ & 24 \\
\hline$\left(0 ; \infty^{(12)}\right) \triangleleft$ & $H_{2,3}$ & 60 \\
\hline$\left(0 ; \infty^{(8)}\right) \triangleleft$ & $H_{2,4}$ & 24 \\
\hline$\left(0 ; \infty^{(20)}\right) \triangleleft$ & $H_{2,5}$ & 60 \\
\hline$\left(0 ; \infty^{(q)}\right) \triangleleft$ & $H_{2, q}$ & $2 q$ \\
\hline$\left(0 ; \infty^{(6)}\right) \triangleleft$ & $H_{3,3}$ & 12 \\
\hline$\left(0 ; \infty^{(12)}\right) \triangleleft$ & $H_{3,4}$ & 24 \\
\hline$\left(0 ; \infty^{(30)}\right) \triangleleft$ & $H_{3,5}$ & 60 \\
\hline$\left(0 ; \infty^{(p)}\right) \triangleleft$ & $H_{p, p}$ & $p$ \\
\hline
\end{tabular}


Corollary 2. The number of normal genus 0 torsion-free subgroups of generalized Hecke groups is;

4 if $p=2$ and $q=3$

2 if $p=2$ and $q=4$ or 5

1 if $p=2$ and $q>5$

2 if $p=3$ and $q=3$

1 if $p=3$ and $q=4$ or 5

1 if $p=q>3$

\section{Even Subgroups of $H_{p, q}$}

In this section, we give an important normal subgroup of the generalized Hecke groups $H_{p, q}$, for even values of $p$ and $q$, called even subgroup. We use the matrix representation $\left(\begin{array}{ll}a & b \\ c & d\end{array}\right)$ for the transformation $\frac{a z+b}{c z+d}$ in the rest of our study.

In [16], Calta and Schmidt defined two types of elements of generalized Hecke groups $H_{p, q}$;

$$
\left(\begin{array}{cc}
a & b \lambda_{q} \\
c \lambda_{q} & d
\end{array}\right) \text { and }\left(\begin{array}{cc}
a \lambda_{q} & b \\
c & d \lambda_{q}
\end{array}\right)
$$

where $a, b, c, d$ are elements of the trace field $K_{p, q}=\mathbb{Q}\left(\lambda_{p} / 2, \lambda_{q} / 2\right)$. These elements are named even and odd respectively. Multiplication of two same type of elements is even and multiplication of two different types is odd. The matrix representations of the generators of $H_{p, q}$ are;

$$
X=\left(\begin{array}{cc}
0 & -1 \\
1 & (1-b) \lambda_{q}
\end{array}\right) \text { and } Y=\left(\begin{array}{cc}
0 & -1 \\
1 & -\lambda_{q}
\end{array}\right)
$$

with $b=\frac{\lambda_{p}+\lambda_{q}}{\lambda_{q}}$. These generators are odd type. As a consequence; every element of the generalized Hecke group $H_{p, q}$ is one of the two types as it can be represented as a product of powers of the generators $X$ and $Y$. We denote the set of all event types of elements as $H_{E_{p, q}}$, i.e.;

$$
H_{E_{p, q}}=\left\{E=\left(\begin{array}{cc}
x & y \lambda_{q} \\
z \lambda_{q} & w
\end{array}\right): E \in H_{p, q}\right\}
$$

It is obvious that the set $H_{E_{p, q}}$ forms a subgroup of $H_{p, q}$. The group $H_{p, q}$ is equal to union of two cosets; 


$$
H_{p, q}=H_{E_{p, q}} \cup X H_{E_{p, q}}
$$

Since the index is two, $H_{E_{p, q}}$ is a normal subgroup of $H_{p, q}$.

Theorem 5. The even subgroup of $H_{p, q}$ is isomorphic to the free product of infinite cyclic group and two finite cyclic groups of order $p / 2$ and $q / 2$. Furthermore the signature of this subgroup is $\left(0 ; p / 2, q / 2, \infty^{(2)}\right)$.

Proof. Since the order of the quotient group is two, there exists a homomorphism of the group $H_{p, q}$ to the cyclic group of order two, by taking the generators $X$ and $Y$ to the element of order two and $X Y$ to the identitiy. Using the permutation method we have the signature of the even subgroup of generalized Hecke group $H_{p, q}$ as $\left(0 ; p / 2, q / 2, \infty^{(2)}\right)$.

We can have the presentation of even subgroup by Reidemeister-Schreier method. The presentation of the quotient group is;

$$
H_{p, q} / H_{E_{p, q}}=<X, Y: \quad X^{2}=Y^{2}=(X Y)=I>
$$

After choosing the Schreier transversal as $\Sigma=\{I, X\}$ the algorithm follows;

$$
\begin{array}{ll}
I . X .(X)^{-1}=I & I . Y .(X)^{-1}=Y . X^{p-1} \\
X . X .(I)^{-1}=X^{2} & X . Y .(I)^{-1}=X Y
\end{array}
$$

Thus we find the generators from the relation, $\left(Y \cdot X^{p-1}\right) \cdot(X Y)=Y^{2}$, as $X^{2}$, $Y^{2}$ and $X Y$. And we have the presentation;

$H_{E_{p, q}}=<X^{2}, Y^{2}, X Y:\left(X^{2}\right)^{p / 2}=\left(Y^{2}\right)^{q / 2}=(X Y)^{\infty}=I \quad>\simeq C_{p / 2} * C_{q / 2} * \mathbb{Z}$

If at least one of the integers $p$ and $q$ is odd, it is not possible to have an even subgroup.

There is a relation between the even subgroup of the generalized Hecke group $H_{p, q}$ and commutator subgroup $H_{p, q}^{\prime}$. We know from [1] that the commutator subgroup $H_{p, q}^{\prime}$ is a normal subgroup of index $p . q$ of generalized Hecke group $H_{p, q}$. Since the definition of the commutator of two elements, the commutator subgroup $H_{p, q}^{\prime}$ is included in even subgroup $H_{E_{p, q}}$. It is obvious that the index of $H_{p, q}^{\prime}$ in $H_{E_{p, q}}$ is $p . q / 2$. So we have;

Theorem 6. Let $p$ and $q$ be even integers. Then the commutator subgroup $H_{p, q}^{\prime}$ of $H_{p, q}$ is a normal subgroup of the even subgroup $H_{E_{p, q}}$. Furthermore the index is $\left[H_{E_{p, q}}, H_{p, q}^{\prime}\right]=\frac{p \cdot q}{2}$.

Corollary 3. Any subgroup of the commutator subgroup $H_{p, q}^{\prime}$ of generalized Hecke group $H_{p, q}$ consists of only even elements. 
The extended generalized Hecke groups $\bar{H}_{p, q}$ defined in [14] and obtained by adding extra one generator $R=\left(\begin{array}{ll}0 & 1 \\ 1 & 0\end{array}\right)$ of order two, called reflection. So the elements of $\bar{H}_{p, q}$ have determinant \pm 1 . Let $T$ be an element of $\bar{H}_{p, q}$ with determinanat -1 . It is easy to see from the presentation of $\bar{H}_{p, q}$ that there is an element $S$ in $H_{p, q}$ such that $T=R . S$.

The definiton of odd and even elements can be generalized to extended generalized Hecke groups.

Definition 1. We can classify the elements of $\bar{H}_{p, q}$ by; $\left(\begin{array}{cc}a & b \lambda_{q} \\ c \lambda_{q} & d\end{array}\right)$ with $a d-b c \lambda_{q}^{2}= \pm 1$ is called even type
$\left(\begin{array}{cc}a \lambda_{q} & b \\ c & d \lambda_{q}\end{array}\right)$ with $a d \lambda_{q}^{2}-b c \lambda_{q}^{2}= \pm 1$ is called odd type.

All generators of $\bar{H}_{p, q}$ are odd types from the above definition. Hence we can obtain the even subgroup $\bar{H}_{E_{p, q}}$ of $\bar{H}_{p, q}$ for even values of $p$ and $q$.

Theorem 7. The even subgroup of $\bar{H}_{p, q}$ defined as;

$$
\bar{H}_{E_{p, q}}=\left\{E=\left(\begin{array}{cc}
x & y \lambda_{q} \\
z \lambda_{q} & w
\end{array}\right): E \in \bar{H}_{p, q}\right\}
$$

is a normal subgroup of $\bar{H}_{p, q}$ of index 2 . Moreover;

$$
\bar{H}_{p, q}=\bar{H}_{E_{p, q}} \cup X \bar{H}_{E_{p, q}}
$$

and the generators of $\bar{H}_{E_{p, q}}$ are $X^{2}, X Y, Y X^{p-1}$ and $X R$.

Proof. It is obvious from the definitions of odd and even types of elements that the index is 2. Since $X$ is an odd element, then $X \notin \bar{H}_{E_{p, q}}$. So we have the other coset $X \bar{H}_{E_{p, q}}$. Now our aim is to find the generators of $\bar{H}_{E_{p, q}}$ by using the Reidemeister-Schreier method. Let us construct the Schreier transversal as $\Sigma=\{I, X\}$. Then all possible products are listed below;

$$
\begin{array}{ll}
I . X .(X)^{-1}=I & X . X .(I)^{-1}=X^{2} \\
\text { I.Y. }(X)^{-1}=Y X^{p-1} & X . Y .(I)^{-1}=X Y \\
\text { I.R. }(X)^{-1}=R X^{p-1} & \text { X.R. }(I)^{-1}=X R
\end{array}
$$

From the relations of the generators of $\bar{H}_{p, q}$ all generators of the even subgroup $\bar{H}_{E_{p, q}}$ are $X^{2}, X Y, Y X^{p-1}, X R$.

Now we want to state the relation between the commutator subgroup $\bar{H}_{p, q}^{\prime}$ and the even subgroup $\bar{H}_{E_{p, q}}$. For even values of $p$ and $q$ the commutator 
subgroup $\bar{H}_{p, q}^{\prime}$ is isomorphic to the product $C_{p / 2} * C_{p / 2} * C_{q / 2} * C_{q / 2} * \mathbb{Z}[1]$. $\bar{H}_{p, q}^{\prime}$ consists of even types of elements because of the similar reason for $H_{p, q}$.

Theorem 8. Let $p$ and $q$ be even integers. Then the commutator subgroup $\bar{H}_{p, q}^{\prime}$ of $\bar{H}_{p, q}$ is a normal subgroup of the even subgroup $\bar{H}_{E_{p, q}}$. Furthermore the index is $\left[\bar{H}_{E_{p, q}}, \bar{H}_{p, q}^{\prime}\right]=4$

Proof. Since all commutators is even type, the inclusion is obvious. We know from [1] the index of $\bar{H}_{p, q}^{\prime}$ in $\bar{H}_{p, q}$ is 8 . Then the required index is 4 .

\section{References}

[1] Ş. Kaymak, B. Demir, Ö. Koruoğlu, R. Sahin, Commutator Subgroups of Generalized Hecke and Extended Generalized Hecke Groups. Submitted.

[2] I.N. Cangul and O. Bizim, Normal Subgroups of Hecke Groups on Sphere and Torus, Tr. J. of Mathematics, 22, 369-377, (1998).

[3] I.N. Cangul, Normal Subgroups of Hecke Groups, Ph.D. Thesis, Southampton University, (1993).

[4] I.N. Cangul, Normal Subgroups and Elements of $H^{\prime}\left(\lambda_{q}\right)$, Tr. J. of Mathematics, 23, 251-256, (1999).

[5] I.N. Cangul, O. Bizim, Commutator Subgroups of Hecke Groups, Bull. Inst. Math. Acad. Sinica, 30, 253-259, (2002).

[6] R. Sahin, O. Bizim, Some Subgroups of Extended Hecke Groups $\bar{H}\left(\lambda_{q}\right)$, Actua. Math. Sci., 23,(4), 497-502, (2003).

[7] R. Sahin, O. Bizim, I.N. Cangul, Commutator Subgroups of the Extended Hecke Groups $\bar{H}\left(\lambda_{q}\right)$, Czech. Math., 28, 253-259, (2004).

[8] E. Hecke, Über die Bestimmung Dirichletscher Reihen durch ihre Funktionalgleichung, Math. Ann. 112, 664-699, (1936).

[9] D. Singerman, Finitely Maximal Fuchsian Groups, J. London Math. Soc., (2), 6, 29-38, (1972).

[10] I.N. Cangul and D. Singerman, Normal Subgroups of Hecke Groups and Regular Maps, Math. Proc. Camb. Phil. Soc. 123, 59, (1998).

[11] Lehner, J. and Newman, M., Real Two-Dimensional Representations of the Modular Group and Related Groups, .Amer. J. Math. 87, 945-954, (1965). 
[12] Lehner, J. and Newman, M., Real Two-Dimensional Representations of the Free Product of Two Finite Cyclic Groups, Proc. Camb. Phil. Soc. 62, 135, (1965).

[13] J. Lehner, Uniqueness of a class of Fuchsian groups, III. J. Math. Surveys, 8, A.M.S. Providence, R.L. (1964).

[14] B. Demir, Ö. Koruoğlu, R. Sahin, Conjugacy Classes of Extended Generalized Hecke Groups. Submitted.

[15] K. Calta and T. A. Schmidt, Continued fractions for a class of triangle groups, J. Aust. Math. Soc. 93, No. 1-2, 21-42 (2012).

[16] K. Calta and T. A. Schmidt, Infinitely many lattice surfaces with special pseudo-Anosov maps, J. Mod. Dyn. 7, No. 2, 239-254 (2013).

Bilal Demir,

Necatibey Faculty of Education, Department of Secondary Mathematics Education,

Balıkesir University,

10100 Balıkesir, Turkey,

Email: bdemir@balikesir.edu.tr

Özden Koruoğlu,

Necatibey Faculty of Education, Department of Secondary Mathematics Ed-

ucation,

Balıkesir University,

10100 Balıkesir, Turkey,

Email: ozdenk@balikesir.edu.tr

Recep Sahin,

Faculty of Science and Arts, Department of Mathematics,

Balıkesir University,

10145 Çă̆ış Campus, Balıkesir, Turkey

Email: rsahin@balikesir.edu.tr 